\title{
Reduction of pancreatic cancer cell viability and induction of apoptosis mediated by siRNA targeting DNMT1 through suppression of total DNA methyltransferase activity
}

\author{
MIN XU $^{1 *}, J_{U N}$ GAO $^{1 *}$, YI QI DU ${ }^{1 *}$, DAO-JIAN GAO ${ }^{1}$, YU QI ZHANG ${ }^{1}$, ZHAO SHEN LI $^{1}$, \\ YOU LI ZHANG ${ }^{2}$, YAN FANG GONG ${ }^{1}$ and PING XU ${ }^{2}$ \\ ${ }^{1}$ Department of Gastroenterology, Changhai Hospital, The Second Military Medical University, Shanghai 200433; \\ ${ }^{2}$ Department of Gastroenterology, Jiangsu University Affiliated Hospital, Jiangsu 212001, P.R. China
}

Received December 17, 2009; Accepted May 6, 2010

DOI: $10.3892 / \mathrm{mmr} 00000320$

\begin{abstract}
Aberrant methylation leads to epigenetic changes in human genes that may cause carcinogenesis. DNA methyltransferase 1 (DNMT1) plays an important role in maintaining DNA methylation patterns during genomic DNA replication. To understand the role of this protein in pancreatic cancer cell growth and apoptosis, small interfering RNA (siRNA) oligonucleotides were used to knockdown DNMT1 expression in pancreatic cancer PaTu8988 cells. We found that the DNMT1 siRNA markedly decreased DNMT1 expression and total DNA methyltransferase activity in the cells. Upon the inhibition of DNMT1 expression, the proliferation of the tumor cells was inhibited. Tumor cell growth was arrested in the S-phase of the cell cycle and cells underwent apoptosis. The expression of p21 was up-regulated and the ratio of Bax/ Bcl-2 expression was increased after DNMT1 knockdown in PaTu8988 cells. Furthermore, DNMT1 siRNA caused demethylation of the tumor suppressor gene hMLH1, resulting in its re-expression in PaTu8988 cells. The results of this study suggest that DNMT1 siRNA oligonucleotides are candidates for further evaluation as therapeutic tools for the clinical control of pancreatic cancer.
\end{abstract}

\section{Introduction}

Epigenetic regulation of gene expression mediated through DNA cytosine methylation, histone modifications and chromatin remodeling plays a critical role in human cell

Correspondence to: $\mathrm{Dr}$ Zhaoshen Li, Department of Gastroenterology, Changhai Hospital, The Second Military Medical University, Shanghai 200433, P.R. China

E-mail: lizhaoshen111@gmail.com

\section{*Contributed equally}

Key words: DNA methylation, DNA methytransferase 1, small interfering RNA, pancreatic cancer homeostasis, including cell proliferation, differentiation and apoptosis $(1,2)$. As is the case with alterations in gene coding (such as mutations and deletions), changes in methylation alter gene expression and lead to human tumorigenesis, particularly in the promoter regions of tumor suppressor genes $(1,3)$. Thus, the alteration of gene promoter methylation may lead to novel strategies for the treatment of cancer.

DNA methyltransferases, including DNMT1, DNMT3A and DNMT3B, catalyze the transfer of methyl groups from S-adenosyl-L-methionine (Ado-Met) to cytosine position 5; methylation in promoter regions silences gene expression. DNMT1 plays an important role in the maintenance of genomic DNA methylation during DNA replication, whereas DNMT3A and DNMT3B methylate de novo synthesized DNA to regulate gene expression (4). DNMT1 preferentially acts on hemimethylated $\mathrm{CpG}$ substrates and is involved in the maintenance of specific DNA methylation patterns during DNA replication $(5,6)$. A number of studies have demonstrated that the expression of DNMT1 is significantly elevated in cancers of the breast, colon, endometrium, prostate and stomach, as well as in uterine leiomyomata (7-12).

The inhibition of DNMT1 expression results in the suppression of cell growth in various cancer cell lines in vitro and in vivo (13-18). The suppression of DNMT1 activity using selective inhibitors induces demethylation and re-expression of silenced tumor suppressor genes. DNMT1 has therefore been proposed as a target for anticancer therapy (19). However, recent studies showed that the targeted deletion of DNMT1 by homologous recombination was not sufficient to cause promoter demethylation and gene re-expression in the colon cancer cell line HCT116 (20,21). Deletion of both DNMT1 and DNMT3B reduced overall genomic methylation by $95 \%$ and caused the re-expression of multiple genes, resulting in substantial growth inhibition in HCT116 cells (22). These studies clearly indicated either that these DNMTs compensate for each other, or that their functions overlap.

To further understand the role of DNMT1 in the growth and apoptosis of human cancers (23), we initiated the present study using pancreatic cancer cells. DNMT1 small interfering RNA (siRNA) oligonucleotides were designed and constructed, and the effects of DNMT1 silencing in cells from 
Table I. Primers used in the study.

\begin{tabular}{lllr}
\hline Primer & \multicolumn{1}{c}{ Sense } & \multicolumn{1}{c}{ Antisense } & Size (bp) \\
\hline DNMTl & 5'-GTGGGGGACTGTGTCTCTGT-3' & 5'-TGAAAGCTGCATGTCCTCAC-3' & 204 \\
bcl-2 & 5'-TCCATGTCTTTGGACAACCA-3' & 5'-CTCCACCAGTGTTCCCATCT-3' & 203 \\
Bax & 5'-TGTCTGTCTTGTCCCCTTCC-3' & 5'-ACCTTGAGCACCAGTTTGCT-3' & 301 \\
p21 & 5'-TTAGCAGCGGAACAAGGAGT-3' & 5'-AGAAACGGGAACCAGGACAC-3' & 252 \\
hMLH1 & 5'-TCCCGAAAGGAAATGACTGC-3' & 5'-CTCCGATAACCTGAGAACACCAAA-3' & 279 \\
GAPDH & 5'-GCACCGTCAAGGCTGAGAAC-3' & 5'-ATGGTGGTGAAGACGCCAGT-3' & 142 \\
hMLH1 $^{\text {a }}$ & 5'-GGAGTGAAGGAGGTTAYGGGTAAGT-3' & 5'-AAAAACRATAAAACCCTATACCTAATCT-3' & 182
\end{tabular}

aPrimer used for bisulfate-specific PCR.

the pancreatic cancer cell line PaTu8988 were determined. The effects of siRNA on DNMT activity and then on cell proliferation, apoptosis and gene expression were evaluated, particularly the expression of the tumor suppressor gene hMLH1.

\section{Materials and methods}

Cell culture and gene transfection. The pancreatic adenocarcinoma cell line PaTu8988 was a kind gift from Professor Thomas Elsaesser of Philipps University, Marburg, Germany. PaTu8988 cells were cultured in 96- or 6-well tissue culture plates in Dulbecco's modified Eagle's medium (DMEM; Invitrogen, Carlsbad, CA, USA) containing 10\% fetal bovine serum (FBS; Invitrogen), penicillin $(1 \mathrm{U} / \mathrm{ml})$ and streptomycin $(50 \mathrm{mg} / \mathrm{l})$ at $37^{\circ} \mathrm{C}$ and $5 \% \mathrm{CO}_{2}$. For gene transfection, the cells were seeded and grown overnight and then transfected with siRNA or negative control siRNA oligonucleotides (Ambion, Austin, TX, USA) at 15 or $30 \mathrm{nmol} / \mathrm{l}$ using Lipofectamine 2000 (Invitrogen) in Opti-DMED-reduced serum medium (Invitrogen). The cell culture medium was refreshed after $6 \mathrm{~h}$ with complete growth medium and the cells were incubated for an additional $48 \mathrm{~h}$. RNA and protein from these cells were then extracted for further analysis. The siRNA sequences used to target DNMT1 were 5'-GGC GGC UCA AAG AUU UGG Att-3' (sense) and 5'-UCC AAA UCU UUG AGC CGC Ctg-3' (antisense), and were designed based on GenBank accession no. NM_001379.

Quantitative real-time reverse transcription-polymerase chain reaction. Real-time reverse transcription-polymerase chain reaction (RT-PCR) was performed to detect the expression of the genes DNMT1, Bax, Bcl-2, p21, hMLH1 and GAPDH. The primers used are listed in Table I. RT-PCR was carried out with a real-time PCR Master Mix (Toyobo, Japan) according to the manufacturer's instructions. Quantitative real-time PCR analysis was performed in a 7500 Fast Real-Time PCR device (Applied Biosystems, Foster City, CA, USA). The amplification conditions were initial denaturation at $95^{\circ} \mathrm{C}$ for $1 \mathrm{~min}$, denaturation at $95^{\circ} \mathrm{C}$ for $15 \mathrm{sec}$, annealing at $60^{\circ} \mathrm{C}$ for $15 \mathrm{sec}$ and elongation at $72^{\circ} \mathrm{C}$ for $45 \mathrm{sec}$ for 40 cycles. The melting curves at 60 and $95^{\circ} \mathrm{C}$ were obtained following the manufacturer's instructions. The relative quantity (RQ) was determined using the $2^{(-\Delta \Delta C t)}$ method. GAPDH mRNA was used as a control and each experiment was performed in duplicate.
Protein extraction and Western blot analysis. Total cellular protein was extracted from cells using an M-PER mammalian protein extraction buffer (Pierce, Rockford, IL, USA) with $0.5 \mathrm{mM}$ PMSF. The protein samples were boiled at $95^{\circ} \mathrm{C}$ for $5 \mathrm{~min}$, centrifuged at $12,000 \mathrm{rpm}$ for $10 \mathrm{~min}$, subjected to sodium dodecyl sulfate polyacrylamide gel electrophoresis (SDS-PAGE, 12\%) and electronically transferred onto PVDF membranes (Millipore, Billerica, MA, USA). The membranes were blocked in a blocking buffer containing $5 \%$ non-fat milk powder and $0.1 \%$ Tween-20 in phosphate-buffered saline (PBS) for $2 \mathrm{~h}$ at room temperature. The membranes were next incubated with anti-DNMT1 (Abcam, Cambridge, MA, USA) or anti- $\beta$-actin (Sigma, St. Louis, MO, USA) for $90 \mathrm{~min}$ and then washed three times for 15 min each in PBS containing 5\% non-fat milk powder and $0.1 \%$ Tween-20. The membranes were further incubated with an anti-rabbit or anti-mouse secondary antibody $(1: 1,000)$ for $2 \mathrm{~h}$ at room temperature. The target protein bands were detected with ECL chemiluminescence reagent (Pierce).

Water-soluble tetrazolium salt- 8 assay. Cell viability was detected using a water-soluble tetrazolium salt-8 (WST-8) assay. Briefly, the cells were grown and transfected with DNMT1 or negative control siRNA oligonucleotides. After $48 \mathrm{~h}, 10 \mu \mathrm{l}$ of the WST- 8 reagent was added to each well and the cells were incubated for an additional $4 \mathrm{~h}$. Absorbance was measured at $450 \mathrm{~nm}$ with an ELISA plate reader (Thermo ELISA Reader, USA).

Flow cytometry assay. Apoptosis levels were detected by flow cytometry with an Annexin V-FITC kit (Jingmei Biotech Company, Shanghai, P.R. China). Briefly, the cells were grown and transfected with DNMT1 or negative control siRNA oligonucleotides. After $48 \mathrm{~h}$, the cells were washed twice with PBS and incubated with $4 \mu \mathrm{l}$ of Annexin $\mathrm{V}$ incubation reagent in the dark for $15 \mathrm{~min}$ at room temperature. Subsequently, $300 \mu \mathrm{l}$ of binding buffer was added and the samples were analyzed on a FACSCalibur flow cytometer (Becton Dickinson, Franklin Lakes, NJ, USA) equipped with Cell Quest Pro software. The data were analyzed using ModFit LT v. 3.1 software (Verity Software House, www.vsh.com).

Cell cycle analysis. Cell cycle analysis was performed using propidium iodide (PI) staining and flow cytometric analysis. 
The cells were grown and transfected with DNMT1 or negative control siRNA oligonucleotides. After 48 h, 1x10 cells were washed twice in ice-cold PBS and fixed in $70 \%$ ethanol (v/v) at room temperature for at least $30 \mathrm{~min}$, and then stored at $-20^{\circ} \mathrm{C}$ overnight. The next day, the cells were washed twice with PBS and stained with PI $(50 \mu \mathrm{g} / \mathrm{ml})$. DNase-free RNase $(10 \mathrm{mg} /$ $\mathrm{ml}$; Bachem California, Torrance, CA, USA) was added and the samples were incubated for $30 \mathrm{~min}$ before flow cytometric analysis. Red fluorescence was measured with a FACSCalibur flow cytometer (Becton Dickinson) and the data were analyzed using ModFit LT v. 3.1 software (Verity Software House).

DNA methyltransferases activity assay. Total DNA methyltransferase activity was measured using the EpiQuik ${ }^{\mathrm{TM}}$ DNA Methyltransferase Assay kit (Epigentek, Brooklyn, NY, USA) as previously described (19). Briefly, the cells were grown and transfected with DNMT1 or negative control siRNA oligonucleotides. After $48 \mathrm{~h}$, the nuclear extract was isolated from the cells using the EpiQuik Nuclear Extraction kit (Epigentek). Subsequently, $3 \mu \mathrm{g}$ of the nuclear extract were incubated with a methylation substrate for $1 \mathrm{~h}$ at $37^{\circ} \mathrm{C}$ and exposed to the capture antibody for $60 \mathrm{~min}$ and the detection antibody for $30 \mathrm{~min}$ at room temperature. Absorbance was then determined using a microplate reader at $450 \mathrm{~nm}$. Data are represented as the relative activity of the untreated cells with the basal DNMT activity set to $100 \%$.

Bisulfite-specific ploymerase chain reaction based cloning and sequencing of the hMLH1 gene. The methylation status of the hMLH1 gene was monitored using a bisulfate-specific PCR (BSP). The cells were grown and transfected with DNMT1 or negative control siRNA oligonucleotides. After $48 \mathrm{~h}$, the genomic DNA was extracted from the cells using a standard proteinase $\mathrm{K}$ and phenol-chloroform extraction protocol. Bisulfite modification was performed with a Gold EZ DNA Methylation-Gold kit ${ }^{\mathrm{TM}}$ (Zymed, San Francisco, CA, USA) according to the manufacturer's instructions. The hMLH1 gene promoter (-689 to $-507 \mathrm{bp}$ ) was amplified under previously described conditions (20). The BSP primers used are shown in Table I. PCR products were then cloned into the pMD-18-T vector (TaKaRa, Dalian, P.R. China), amplified and sequenced ( $\sim 10$ clones from each sample were randomly selected for DNA sequencing).

Statistical analysis. Data are summarized as the means \pm SD if appropriate. The values were compared and differences between the means of the DNMT1 or negative control siRNA oligonucleotides were determined using a t-test or ANOVA. A P-value of $<0.05$ was considered statistically significant. Correlation analysis was performed using linear regression and the Pearson's correlation coefficient (R). SPSS software for Windows 13.0 was used to determine the P-values.

\section{Results}

Suppression of DNMT1 expression and reduction of total DNA methyltransferase activity by DNMT1 siRNA. After transfection with DNMT1 siRNA oligonucleotides at concentrations of 15 or $30 \mathrm{nM}$, the levels of DNMT1 mRNA (Fig. 1A) and protein (Fig. 1B) were significantly suppressed in pancreatic
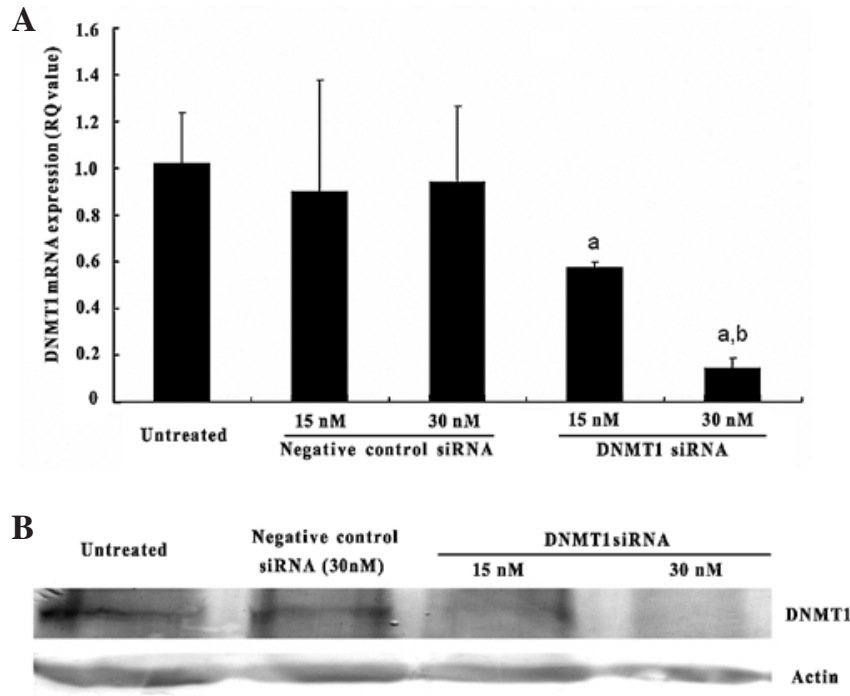

Figure 1. DNMT1 siRNA treatment inhibits DNMT1 mRNA and protein expression. (A) Real-time RT-PCR for analysis of DNMT1 mRNA expression. Pancreatic cancer PaTu8988 cells were grown and transfected with DNMT1 or negative control siRNA oligonucleotides After 48 h, RNA from these cells was isolated and subjected to real-time RT-PCR analysis of DNMT1 mRNA expression. (B) Western blotting was used to analyze DNMT1 protein expression. Pancreatic cancer PaTu8988 cells were grown and transfected with DNMT1 or negative control siRNA oligonucleotides. After $48 \mathrm{~h}$, total cellular protein from these cells was extracted and subjected to Western blot analysis of DNMT1 expression. All experiments were repeated three times. ${ }^{\text {a }}<0.05$ compared to the negative control siRNA; ${ }^{\mathrm{b}} \mathrm{P}<0.05$ compared to $15 \mathrm{nM}$ of DNMT1 siRNA transfection.

cancer PaTu8988 cells using RT-PCR and Western blot analysis, respectively, while the negative control siRNA showed only a slight reduction in DNMT1 expression compared to the untransfected cells. Upon DNMT1 knockdown, the level of total DNMT activity in the cell was significantly lower than that in untransfected cells or in the negative control siRNAtransfected cells (Fig. 2A). Correlation analysis showed a significant association between DNMT1 mRNA levels and DNMT activity in the untransfected cells, negative control siRNA and DNMT1-transfected cells $(\mathrm{R}=0.69, \mathrm{P}=0.004)$ (Fig. 2B). These data demonstrated that DNMT1 contributes significantly to the DNMT activity in PaTu8988 cells.

Effects of DNMT1 siRNA on cell viability, the cell cycle and apoptosis. As shown in Fig. 3A, tumor cell viability was reduced after transfection with DNMT1 siRNA compared to untransfected cells and the negative control siRNA-transfected cells. Cell cycle analysis showed that treatment with DNMT1 siRNA increased the number of cells in the S-phase and reduced the number of cells in the $G_{2}$ phase compared to the untransfected cells and to the cells transfected with negative control siRNA (Fig. 3B).

Flow cytometric analysis was performed to determine whether DNMT1 siRNA induces apoptosis. As shown in Fig. 4A, the percentage of cells that underwent apoptosis was significantly increased in DNMT1 siRNA-transfected cells compared to untransfected and the negative control siRNA-transfected tumor cells. Moreover, the expression ratio of the pro- and anti-apoptotic proteins, Bax and Bcl-2, was increased in cells transfected with DNMT1 siRNA compared 

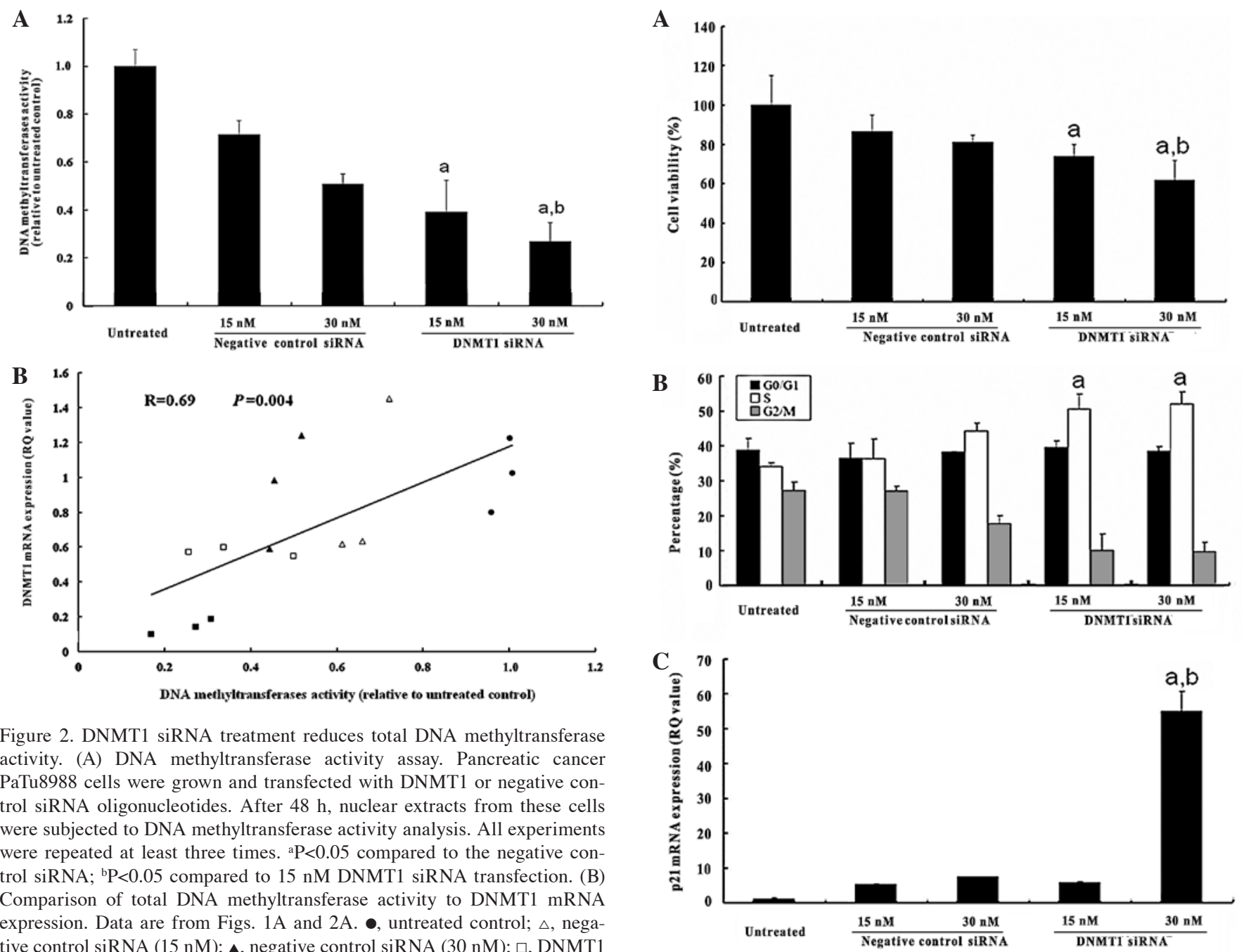
activity. (A) DNA methyltransferase activity assay. Pancreatic cancer PaTu8988 cells were grown and transfected with DNMT1 or negative control siRNA oligonucleotides. After $48 \mathrm{~h}$, nuclear extracts from these cells were subjected to DNA methyltransferase activity analysis. All experiments were repeated at least three times. ${ }^{a} \mathrm{P}<0.05$ compared to the negative control siRNA; ${ }^{b} \mathrm{P}<0.05$ compared to $15 \mathrm{nM}$ DNMT1 siRNA transfection. (B) Comparison of total DNA methyltransferase activity to DNMT1 mRNA expression. Data are from Figs. 1A and 2A. $\bullet$, untreated control; $\Delta$, negative control siRNA (15 nM); $\mathbf{\Delta}$, negative control siRNA (30 nM); $\square$, DNMT1 siRNA (15 nM); $\mathbf{- 1}$ DNMT1 siRNA (30 nM).

to control cells (Fig. 4B). Although the expression of Bcl-2 mRNA was not significantly changed, Bax mRNA expression was increased in DNMT1 siRNA-transfected tumor cells compared to the expression in untransfected and the negative control siRNA-transfected tumor cells.

Using RT-PCR, we showed that p21 expression was up-regulated in cells transfected with DNMT1 siRNA compared to cells transfected with the negative control siRNA (Fig. 3C).

DNMT1 siRNA induces demethylation of the tumor suppressor gene hMLH1. In the PaTu8988 cells, the promoter of the tumor suppressor gene hMLH1 was determined to be methylated using bisulfite-specific PCR-based cloning and sequencing (Fig. 5A). After DNMT1 siRNA transfection, a number of the methylated CpG sites in the hMLH1 gene promoter became unmethylated, and the expression of hMLH1 was significantly up-regulated (Fig. 5B).

\section{Discussion}

In the present study, we determined the effects of DNMT1 knockdown in pancreatic cancer PaTu8988 cells. The inhibition of DNMT1 expression was found to reduce tumor cell

viability, increase the number of cells in the S-phase of the cell cycle and induce apoptosis. Total DNMT activity was reduced in cells treated with DNMT1-specific siRNA, whereas the expression of p21 and Bax was up-regulated compared to untransfected and the negative control siRNA-transfected tumor cells. Furthermore, treatment with DNMT1 siRNA resulted in the demethylation of CpG sites in the hMLH1 gene promoter, which resulted in the re-expression of hMLH1. hMLH1 is a tumor suppressor gene that plays a role in DNA mismatch repair.

DNA methylation is an important epigenetic regulator of gene expression. Several DNA methyltransferases have been identified, including DNMT1, DNMT3A and DNMT3B. 
A

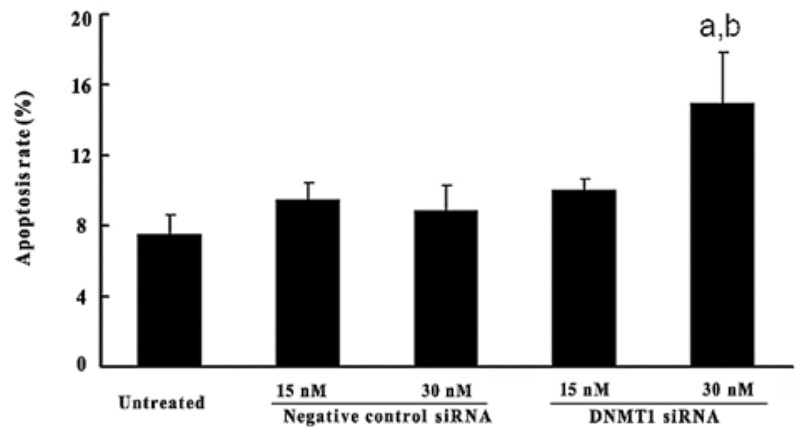

B

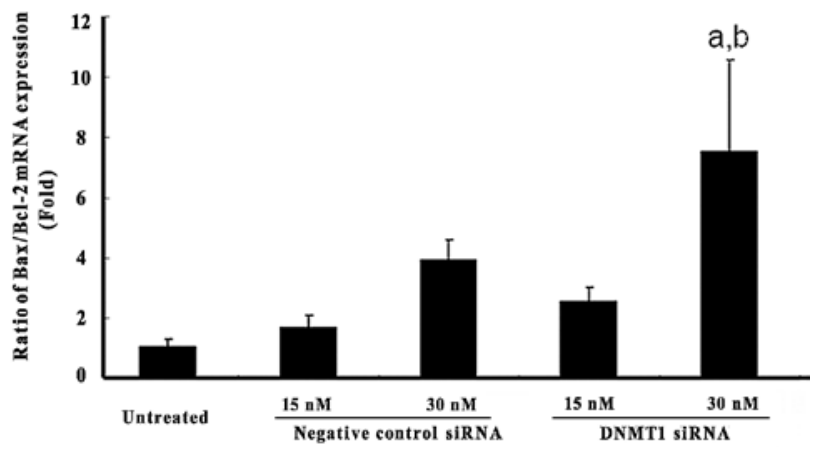

Figure 4. Effects of DNMT1 siRNA treatment on apoptosis and the expression ratio of Bax/Bcl-2. (A) Annexin V and flow cytometry assay. Pancreatic cancer PaTu8988 cells were grown and transfected with DNMT1 or negative control siRNA oligonucleotides. After $48 \mathrm{~h}$, the cells were stained with Annexin V and analyzed by flow cytometry. (B) Real-time RT-PCR for the analysis of Bax and Bcl-2 expression. Pancreatic cancer PaTu8988 cells were grown and transfected with DNMT1 or negative control siRNA oligonucleotides. After 48 h, RNA was isolated and subjected to real-time RT-PCR analysis of Bax and Bcl-2 expression. All experiments were repeated three times. ${ }^{a} \mathrm{P}<0.05$ compared to the negative control siRNA; ${ }^{\mathrm{b}} \mathrm{P}<0.05$ compared to $15 \mathrm{nM}$ DNMT1 siRNA transfection.

These methyltransferases methylate the $\mathrm{CpG}$ islands of gene promoters; this methylation regulates gene expression, which in turn affects human development, growth and homeostasis. Alterations of these methyltransferases were reported to be associated with human tumorigenesis $(25,26)$. For example, the overexpression of DNMT1 or DNMT3B increases the total DNMT activity and prompts the hypermethylation of $\mathrm{CpG}$ islands, resulting in the transcriptional silencing of different tumor suppressor genes (22).

Our results confirmed previous reports that DNMT1 controls a large portion of global DNA methylation in addition to gene-specific methylation in human cancer cells (24), although previous studies showed that the knockout of DNMT1 was not sufficient to cause promoter demethylation and gene re-expression $(20,25)$. This discrepancy suggests that the silencing and expression of tumor suppressor genes may differ in different tumor cell lines. DNMT1 may be involved in the methylation of different genes in different cancer types (29).

Previous studies have shown that the inhibition of DNMT1 leads to the inhibition of the initiation of DNA replication and triggers intra-S-phase cell cycle arrest in various tumor cells $(16,26)$. Although the mechanisms responsible for DNA replication inhibition following DNMT1 knockdown remain unclear, changes in the methylation status of the original compared to the newly-synthesized DNA sequences may
A
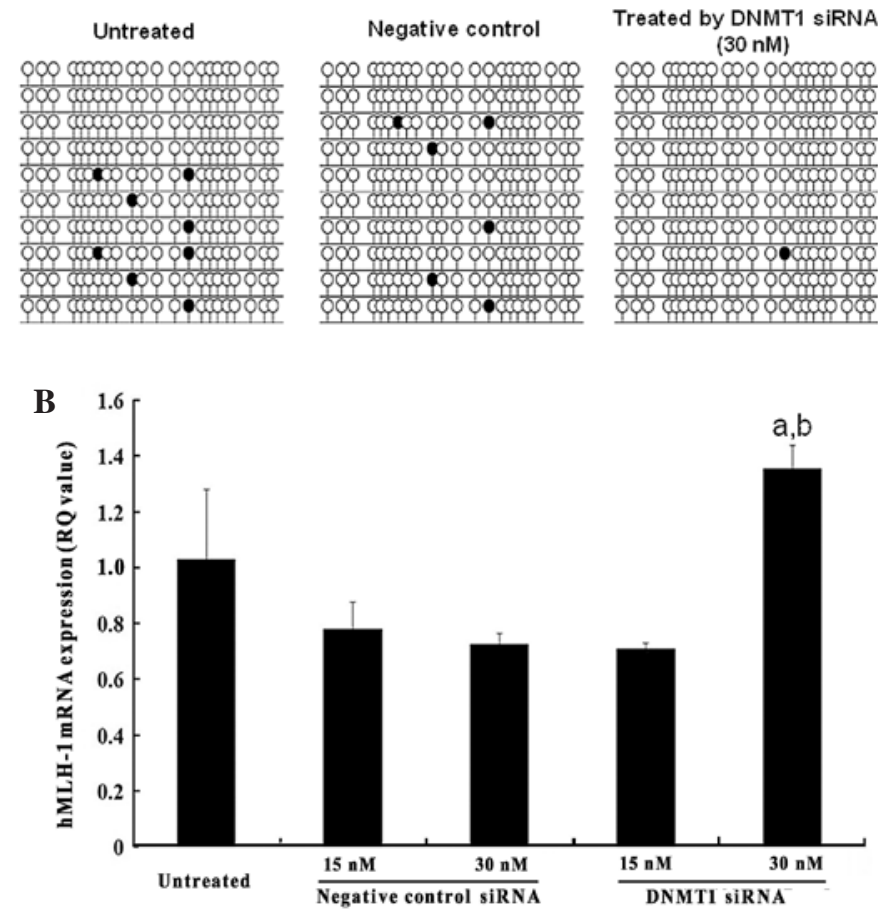

Figure 5. DNMT1 siRNA treatment results in demethylation of the hMLH1 gene promoter and re-expression of hMLH1 mRNA. (A) Bisulfite sequencing analysis. Pancreatic cancer PaTu8988 cells were grown and transfected with DNMT1 or negative control siRNA oligonucleotides. After 48 h, genomic DNA from the cells was isolated and subjected to BSP, DNA cloning, amplification and sequencing. Open circles represent unmethylated $\mathrm{CpG}$ sites; closed circles represent methylated $\mathrm{CpG}$ sites. (B) Real-time RT-PCR to evaluate hMLH1 expression. Pancreatic cancer PaTu8988 cells were grown and transfected with DNMT1 or negative control siRNA oligonucleotides. After $48 \mathrm{~h}$, RNA was isolated and subjected to real-time RT-PCR for the analysis of hMLH1 mRNA expression. All experiments were repeated three times. ${ }^{\mathrm{a}} \mathrm{P}<0.05$ compared to the negative control siRNA; ${ }^{\mathrm{b}} \mathrm{P}<0.05$ compared to $15 \mathrm{nM}$ DNMT1 siRNA transfection.

prevent new DNA synthesis. In our study, we found that treatment with DNMT1 siRNA arrested cells during the S-phase of the cell cycle and inhibited the expression of the cyclindependent kinase inhibitor p21. Our findings are in agreement with previous reports indicating that the inhibition of DNMT activity by a DNMT antagonist or by antisense oligonucleotides results in a rapid up-regulation of the expression of $\mathrm{p} 21$ $(13,17)$.

Apoptosis maintains human cell homeostasis and removes cells when damage is beyond repair. Thus, apoptosis may play a role in preventing carcinogenesis. If a cell is unable to undergo apoptosis due to mutations or biochemical inhibition, it continues to divide. In the present study, DNMT1 siRNA transfection arrested cells at the S-phase of the cell cycle, indicating that DNA replication is halted after DNMT1 suppression. We found that the expression of the pro-apoptosis gene $\mathrm{Bax}$ was up-regulated and that the $\mathrm{Bax} / \mathrm{Bcl}-2$ ratio was increased by DNMT1 siRNA treatment.

Loss of DNA methylation in cells may result in the re-expression of silenced genes. In the present study, the re-expression of MLH1 was induced by DNMT1 siRNA treatment. MLH1 is a single-copy gene involved in the mismatch repair of DNA that is frequently silenced and 
hypermethylated in human colon and gastric cancers $(16,27)$. Re-expression may help maintain DNA integrity. Further study of the effects of re-expression is required, as the re-expression of some genes may be deleterious. Our future investigations will focus on the molecular mechanisms responsible for the effects of DNMT1 siRNA on cell viability. The current data suggest that DNMT1 siRNA has potential in the treatment of pancreatic cancer.

\section{Acknowledgements}

This work was partially supported by the Chinese National Key Technology R\&D Program (Grant No. 2006BAI02A12), the Natural Science Foundation of Jiangsu Province (Grant No. BK2009210), and Jiangsu Province's Health Department Program (Grant No. H200836).

\section{References}

1. Esteller M: Epigenetics in cancer. N Engl J Med 358: 1148-1159, 2008.

2. Robertson KD and Jones PA: DNA methylation: past, present and future directions. Carcinogenesis 21: 461-467, 2000.

3. Chen T and Li E: Structure and function of eukaryotic DNA methyltransferases. Curr Topics Dev Biol 60: 55-89, 2004.

4. Okano M, Bell DW, Haber DA and Li E: DNA methyltransferases dnmt3a and dnmt $3 b$ are essential for de novo methylation and mammalian development. Cell 99: 247-257, 1999.

5. Bestor TH: The DNA methyltransferases of mammals. Hum Mol Gen 9: 2395-2402, 2000.

6. Szyf M, Pakneshan P and Rabbani SA: DNA methylation and breast cancer. Biochem Pharmacol 68: 1187-1197, 2004.

7. Girault I, Tozlu S, Lidereau R and Bieche I: Expression analysis of DNA methyltransferases $1,3 \mathrm{a}$, and $3 \mathrm{~b}$ in sporadic breast carcinomas. Clin Cancer Res 9: 4415-4422, 2003.

8. Li S, Chiang TC, Richard-Davis G, Barrett JC and McLachlan JA: DNA hypomethylation and imbalanced expression of DNA methyltransferases (dnmt1, 3a, and 3b) in human uterine leiomyoma. Gynecol Oncol 90: 123-130, 2003

9. Xiong Y, Dowdy SC, Xue A, et al: Opposite alterations of DNA methyltransferase gene expression in endometrioid and serous endometrial cancers. Gynecol Oncol 96: 601-609, 2005.

10. Agoston AT, Argani P, Yegnasubramanian S, et al: Increased protein stability causes DNA methyltransferase 1 dysregulation in breast cancer. J Biol Chem 280: 18302-18310, 2005.

11. Issa JP, Vertino PM, Wu J, et al: Increased cytosine DNA-methyltransferase activity during colon cancer progression. J Nat Cancer Inst 85: 1235-1240, 1993.

12. Robertson KD, Uzvolgyi E, Liang G, et al: The human DNA methyltransferases (dnmts) 1, 3a and 3b: coordinate mrna expression in normal tissues and overexpression in tumors. Nucleic Acids Res 27: 2291-2298, 1999.
13. Fournel M, Sapieha P, Beaulieu N, Besterman JM and MacLeod AR: Down-regulation of human DNA-(cytosine-5) methyltransferase induces cell cycle regulators p16(ink4a) and p21(waf/cip1) by distinct mechanisms. J Biol Chem 274: 24250-24256, 1999.

14. Ramchandani S, MacLeod AR, Pinard M, von Hofe E and Szyf M: Inhibition of tumorigenesis by a cytosine-DNA, methyltransferase, antisense oligodeoxynucleotide. Proc Nat Acad Sci USA 94: 684-689, 1997.

15. MacLeod AR and Szyf M: Expression of antisense to DNA methyltransferase mrna induces DNA demethylation and inhibits tumorigenesis. J Biol Chem 270: 8037-8043, 1995.

16. Knox JD, Araujo FD, Bigey P, et al: Inhibition of DNA methyltransferase inhibits DNA replication. J Biol Chem 275: 17986-17990, 2000.

17. Milutinovic S, Knox JD and Szyf M: DNA methyltransferase inhibition induces the transcription of the tumor suppressor p21(waf1/cip1/sdi1). J Biol Chem 275: 6353-6359, 2000.

18. Bigey P, Knox JD, Croteau S, Bhattacharya SK, Theberge J and Szyf M: Modified oligonucleotides as bona fide antagonists of proteins interacting with DNA. Hairpin antagonists of the human DNA methyltransferase. J Biol Chem 274: 4594-4606, 1999.

19. Szyf M: DNA methylation properties: Consequences for pharmacology. Trends Pharmacol Sci 15: 233-238, 1994.

20. Rhee I, Jair KW, Yen RW, et al: Cpg methylation is maintained in human cancer cells lacking dnmt1. Nature 404: 1003-1007, 2000.

21. Ting AH, Jair KW, Suzuki H, Yen RW, Baylin SB and Schuebel KE: Cpg island hypermethylation is maintained in human colorectal cancer cells after rnai-mediated depletion of dnmt1. Nat Genet 36: 582-584, 2004.

22. Rhee I, Bachman KE, Park BH, et al: Dnmt1 and dnmt3b cooperate to silence genes in human cancer cells. Nature 416: 552-556, 2002.

23. Sowinska A and Jagodzinski PP: Rna interference-mediated knockdown of dnmt 1 and dnmt3b induces cxcl12 expression in mcf-7 breast cancer and aspc1 pancreatic carcinoma cell lines. Cancer Lett 255: 153-159, 2007.

24. Robert MF, Morin S, Beaulieu N, et al: Dnmt1 is required to maintain cpg methylation and aberrant gene silencing in human cancer cells. Nat Genet 33: 61-65, 2003.

25. Ting AH, Schuebel KE, Herman JG and Baylin SB: Short double-stranded rna induces transcriptional gene silencing in human cancer cells in the absence of DNA methylation. Nat Genet 37: 906-910, 2005.

26. Milutinovic S, Zhuang Q, Niveleau A and Szyf M: Epigenomic stress response. Knockdown of DNA methyltransferase 1 triggers an intra-s-phase arrest of DNA replication and induction of stress response genes. J Biol Chem 278: 14985-14995, 2003.

27. Herman JG, Umar A, Polyak K, et al: Incidence and functional consequences of hmlh1 promoter hypermethylation in colorectal carcinoma. Proc Nat Acad Sci USA 95: 6870-6875, 1998. 